TOPOLOGY ROBUST INTRINSIC SYMMETRIES OF NON-RIGID SHAPES BASED ON DIFFUSION DISTANCES

By

Dan Raviv

Alexander M. Bronstein

Michael M. Bronstein

Ron Kimmel

and

Guillermo Sapiro

IMA Preprint Series \# 2293

( January 2010)

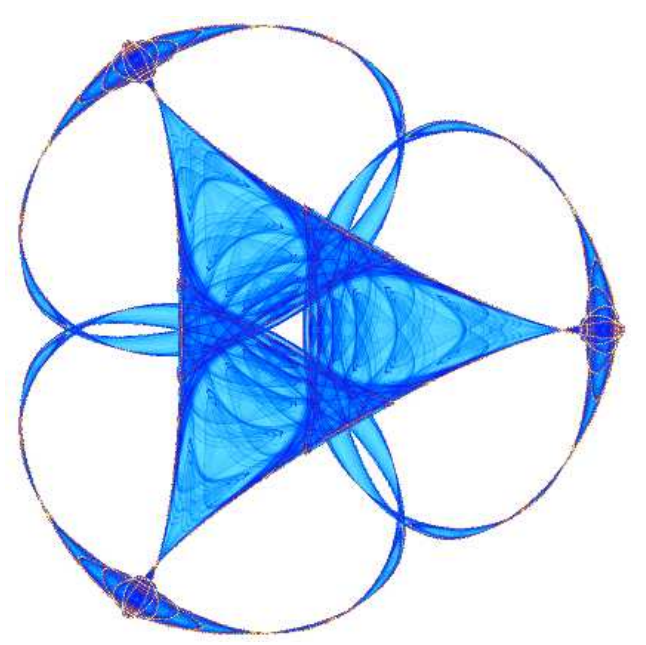

INSTITUTE FOR MATHEMATICS AND ITS APPLICATIONS

UNIVERSITY OF MINNESOTA 400 Lind Hall

207 Church Street S.E.

Minneapolis, Minnesota 55455-0436

Phone: 612-624-6066 Fax: 612-626-7370

URL: http://www.ima.umn.edu 


\title{
Topology Robust Intrinsic Symmetries of non-rigid shapes based on Diffusion Distances Technical Report
}

\author{
$\operatorname{Dan}_{\text {Raviv }} \Omega$ \\ Alexander M. Bronstein $\Omega$ \\ Michael M. Bronstein $\Omega$ \\ Ron Kimmel $^{\Omega}$ \\ Guillermo Sapiro $\Pi$ \\ December 28, 2009
}

\begin{abstract}
Detection and modeling of self-similarity and symmetry is important in shape recognition, matching, synthesis, and reconstruction. While the detection of rigid shape symmetries is well established, the study of symmetries in non-rigid shapes is a much less researched problem. A particularly challenging setting is the detection of symmetries in non-rigid shapes affected by topological noise and asymmetric connectivity. In this paper, we treat
\end{abstract}


shapes as metric spaces, with the metric induced by diffusion distances, and define non-rigid symmetries as self-isometries with respect to the diffusion metric. Experimental results show the advantage of the diffusion metric over the previously proposed geodesic metric for exploring intrinsic symmetries of bendable shapes with possible topological irregularities.

\section{Introduction}

Symmetry or geometric self-similarity plays a fundamental role in Nature and is tightly tied to shape analysis and understanding. The exact definition of symmetry heavily depends on what we understand by the geometry of the shape. Broadly speaking, intrinsic geometry describes the properties of the shape which are invariant to inelastic deformations, while extrinsic geometry is associated with rigid transformations. In the pattern recognition and computer vision literature, there exists a significant number of papers dedicated to finding symmetries in images [29], two-dimensional [1, 2], and three-dimensional shapes $[22,32,34,35]$. A wide spectrum of methods employed for this purpose includes approaches based on dual spaces [13], genetic algorithms [17], moments calculation [10], pair matching [12, 27], and local shape descriptors [47] to name just a few. Traditionally, symmetries are described as extrinsic self-similarity under rotations and reflections. While being adequate for rigid shapes, such a description is inappropriate for non-rigid ones. Extrinsic symmetry can be broken as a result of non-rigid shape deformations, whereas its intrinsic symmetry is preserved 
$[37,38]$.

While finding shape representations that are invariant to bending has been intensively studied in recent years $[5,14,15,18,19,28,30,31,40,42]$, very little has been done in the research of regular surfaces and self-similarity study in non-rigid shapes. Raviv et al. [37] first defined and proposed a computational framework for exploring exact and approximate intrinsic self-similarities based on invariance of geodesic distances. They used the numerical scheme presented in $[5,6]$ for the computation of minimum-distortion correspondence between metric spaces. In [34], Ovsjanikov et al. showed how eigenfunctions of the LaplaceBeltrami operator can be used for identifying reflective intrinsic symmetries. They transformed intrinsic symmetries to (approximate) Euclidean ones in a feature space created by the Laplace-Beltrami eigenfunctions. The reader is also referred to recent papers on such spectral signatures [3, 24, 39, 40, 42].

A major drawback of the mentioned approaches is that topology and connectivity changes (resulting, e.g., from noise and acquisition artifacts) can have a dramatic influence on the intrinsic geometry defined by the geodesic distances. Since the geodesic is the shortest path, even a small change in the topology can cause a significant change in the geodesic metric by affecting the length of many geodesics, thus the approach proposed in [37] is sensitive to topology changes. Ovsjanikov et al. [34] explain why topology noise appears in high frequencies, hence its influence can be reduced in their method. Yet, they can not guarantee robustness to major topology changes.

In $[8,30]$ it was shown that diffusion geometry, arising from the study of heat 
propagation on the surface, can gracefully handle topological and connectivity problems. In this paper, we introduce diffusion symmetries of non-rigid shapes which are robust to topology changes.

The rest of this paper is organized as follows: In Section 2, we present metric models of shapes. In Section 3, we define exact and approximate symmetries, and in Section 4 elaborate on Gromov-Hausdorff distance. Section 5 is dedicated for the numerical framework, followed by Section 6 where we present experimental results. Section 7 concludes the paper.

\section{Shapes as metric spaces}

We model a shape as a metric space $\left(X, d_{X}\right)$, where $X$ is a two-dimensional smooth compact connected and complete Riemannian surface (possibly with boundary) embedded into $\mathbb{R}^{3}$, and $d: X \times X \rightarrow \mathbb{R}_{+} \cup\{0\}$ is a metric measuring distances between pairs of points on $X$. In general, $d$ is independent of $X$ and can be defined quite arbitrarily, provided that it satisfies the metric axioms ,positive definiteness, symmetry, and triangle inequality. There are, however, a few natural choices of $d$ describing different properties of the shape, and giving rise to different geometries. 


\subsection{Euclidean geometry}

Considering $X$ as a subset of the Euclidean space $\mathbb{E}=\mathbb{R}^{3}$, we can measure the distances between points $x$ and $x^{\prime}$ on $X$ using the restricted Euclidean metric,

$$
d_{\mathbb{E}}\left(x, x^{\prime}\right)=\left.d_{\mathbb{E}}\right|_{X \times X}\left(x, x^{\prime}\right) .
$$

We refer to the properties described by $d_{\mathbb{E}}$ as the extrinsic geometry of $X$. Such properties are invariant under Euclidean transformations, translation, rotation, and reflection in $\mathbb{E}$. However, nonrigid deformations may change the extrinsic geometry, and thus the Euclidean metric is not suitable for modeling shapes exhibiting significant deformations.

\subsection{Geodesic geometry}

Geometries invariant to bending, like inelastic deformations which do not stretch or tear the shape, are called intrinsic and are defined using properties of $X$ without resorting to the ambient space $\mathbb{E}$. The simplest example of an intrinsic metric is the geodesic metric, defined as the length of the shortest path on the shape,

$$
d_{X}\left(x, x^{\prime}\right)=\inf _{\gamma \in \Gamma\left(x, x^{\prime}\right)} \ell(\gamma)
$$

where $\Gamma\left(x, x^{\prime}\right)$ is the set of all admissible paths between the points $x$ and $x^{\prime}$ on $X$ and $\ell(\gamma)$ is the length of the path $\gamma^{1}$. The geodesic metric is invariant to inelastic

\footnotetext{
${ }^{1}$ The assumption of completeness implies that $d_{X}$ is realized by a shortest path and thus replace inf with min.
} 
shape deformations, and as a particular case is also invariant to rigid transformations. As a consequence, the geodesic metric is a good choice for describing non-rigid shapes capturing their properties that do not depend on deformations.

\subsection{Diffusion geometry}

Well-known in other fields for decades, diffusion geometry has been popularized in data analysis by Lafon and Coifman $[11,25]$. Informally, the diffusion distances have an effect of averaging over all possible paths connecting two points, while the geodesic distance is the length of just the minimal one. As a consequence, the former is more robust than the geodesic distance in cases where topological changes are present $[33,43]$.

Formally, the diffusion distance, is related to the heat equation,

$$
\left(\Delta_{X}+\frac{\partial}{\partial t}\right) u=0
$$

governing the distribution of heat $u$ on the surface. Here, $\Delta_{X}$ denotes the LaplaceBeltrami operator, a generalization of the Laplacian to non-Euclidean domains. (Note that we define $\Delta_{X}$ as a positive-semidefinite operator, hence its positive sign in (3)). The fundamental solution $h_{t}(x, z)$ of the heat equation (3), also called the heat kernel, is the solution with a point heat source at $x \in X$ used as the initial condition at $t=0$. The heat kernel $h_{t}(x, z)$ describes the heat distribution at time $t$ at point $z \in X$.

For compact manifolds, the Laplace-Beltrami operator has discrete eigende- 
composition of the form

$$
\Delta_{X} \phi_{i}=\lambda_{i} \phi_{i}
$$

where $\lambda_{0}, \lambda_{1}, \ldots$ are eigenvalues and $\phi_{0}, \phi_{1}, \ldots$ are the corresponding eigenfunctions. Using this basis, the heat kernel can be presented as [21]

$$
h_{t}(x, z)=\sum_{i=0}^{\infty} e^{-\lambda_{i} t} \phi_{i}(x) \phi_{i}(z) .
$$

Note that for a shape consistency of a single connected component $\lambda_{0}=0, \phi_{0}=$ const, and $\lambda_{i}>0$.

The diffusion distance is the family of metrics of the form

$$
\begin{aligned}
d_{X, t}^{2}(x, y) & =\left\|h_{t}(x, \cdot)-h_{t}(y, \cdot)\right\|_{L_{2}(X)}^{2} \\
& =\int_{X}\left|h_{t}(x, z)-h_{t}(y, z)\right|^{2} d z \\
& =\sum_{i=1}^{\infty} e^{-2 \lambda_{i} t}\left(\phi_{i}(x)-\phi_{i}(y)\right)^{2}
\end{aligned}
$$

parametrized by the scale $t$. Since the diffusion distance is derived from the Laplace-Beltrami operator which is an intrinsic property of the shape, it is an intrinsic metric and is therefore bending-invariant. Besides that, it shows better robustness to topology changes, as we present in the following. 


\section{Symmetry}

The metric model of shapes allows to formalize the notions of invariance, selfsimilarity, and symmetry. From the metric viewpoint, a shape is invariant under a transformation if the metric structure is unaffected by the transformation. More formally, two shapes $(X, d)$ and $(Y, \tilde{d})$, where $d, \tilde{d}$ are the respective metrics, are isometric if there exists a bijective map $\varphi: X \rightarrow Y$ (called isometry) such that $\tilde{d} \circ(\varphi \times \varphi)=d$. In particular, the shape $(X, d)$ is self-similar or self-isometric if there exists a permutation (bijective map $f$ from $X$ to itself) which is an isometry with respect to $d$, i.e., $d \circ(f \times f)=d$.

It can be easily shown that symmetries form a subgroup of the group of permutations $(\Pi(X), \circ)$, where $\Pi(X)=\{g: X \stackrel{1: 1}{\rightarrow} X\}$ and $\circ$ is the function composition operator. We denote the group of symmetries of $X$ by

$$
\operatorname{Sym}(X, d)=\{f \in \Pi(X): d \circ(f \times f)=d\} .
$$

\subsection{Approximate symmetries}

Because the true symmetry is an idealized rather than naturally occurring phenomenon, a relaxation of the notion of symmetry is required [47]. In the Euclidean case, approximate symmetry can be defined as a non-trivial Euclidean transformation $i$ satisfying $i(X) \approx X$. In the more general setting, the relaxation of the notion of symmetry is possible by allowing $d \circ(f \times f) \approx d$ in the definition of selfisometry. More formally, we say that the shape $(X, d)$ is $\varepsilon$-self-isometric if there 
exists a permutation $f \in \Pi(X)$ with distortion

$$
\begin{aligned}
\operatorname{dis}(f, d) & =\|d \circ(f \times f)-d\|_{\infty} \\
& =\max _{x, x^{\prime} \in X}\left|d\left(x, x^{\prime}\right)-d\left(f(x), f\left(x^{\prime}\right)\right)\right| \leq \varepsilon .
\end{aligned}
$$

We denote the family of all $\varepsilon$-self-isometries of $(X, d)$ by

$$
\operatorname{Iso}_{\varepsilon}(X, d)=\{f \in \Pi(X): \operatorname{dis}(g, f) \leq \varepsilon\} .
$$

In particular, for $\varepsilon=0$ we get the symmetry group $\operatorname{Iso}_{0}(X, d)=\operatorname{Iso}(X, d)$. Note that unlike $\operatorname{Iso}(X, d), \operatorname{Iso}_{\varepsilon}(X, d)$ is not a group for $\varepsilon>0$, since it does not satisfy the closure property: a composition of two $\varepsilon$-self-isometries is in general a $2 \varepsilon$ self-isometry.

\subsection{Local asymmetry}

In many cases, the shape asymmetry is as important as symmetry, as it indicates some abnormality of a shape. Given an $\varepsilon$-symmetric shape and one of its approximate symmetries $f \in \operatorname{Iso}_{\varepsilon}(X, d)$, we can compute the contribution of each point to the shape asymmetry as the local shape asymmetry,

$$
\operatorname{asym}_{(X, d)}(f, x)=\max _{x^{\prime} \in X}\left|d_{X}\left(x, x^{\prime}\right)-d_{X}\left(f(x), f\left(x^{\prime}\right)\right)\right|,
$$

quantifying the distortion of $f$ at a point $x$. Points with large local asymmetry are responsible for symmetry breaking. Global shape asymmetry can be calculated as 
the supremum of all local ones, which is the same as the distortion $\operatorname{dis}(f, d)$.

\subsection{Symmetry space}

Though we cannot use a group structure to represent approximate symmetries, we can still explore the space of permutation $\Pi(X)$, where each function $g$ has its distortion $\operatorname{dis}(g, d)$. If the distortion is a smooth function then choosing local minima as symmetry candidates is the logical thing to do. For smooth mappings this is the case, which means that local minima of distortions are good candidates for significant approximate symmetry. See supporting proof in Appendix A for the continuity of the distortion near true symmetries.

The space of functions $\Pi(X)$ can also be endowed with some standard metric that measures the distance between two permutations $f$ and $g$ on $X$, defined as

$$
d_{\Pi(X)}(f, g)=\max _{x \in X} d(f(x), g(x))=d(f(X), g(X)),
$$

which, in turn, depends on the choice of the metric $d$. We refer to the set

$$
B_{\Pi(X)}(g, r)=\left\{f \in \Pi(X): d_{\Pi(X)}(g, f) \leq r\right\}
$$

as the closed metric ball (intrinsic or extrinsic, according to the choice of the metric in the definition of $d_{\Pi(X)}$ ) of radius $r$ centered at $g$. (We will omit $r$ referring to a ball of some unspecified radius).

Since true symmetries have zero distortion (i.e. $\operatorname{dis}(g, d)=0$ ), they are the 
global minimizers of the distortion function $\operatorname{dis}(g, d)$. Moreover, they are also local minimizers of the distortion, in the sense that for every approximate symmetry $g$, there exists a sufficiently small neighborhood $B_{\Pi(X)}(g)$, such that any $f \in B_{\Pi(X)}(g), \operatorname{dis}(f) \geq \operatorname{dis}(g)$. While trivial for true symmetries, this observation motivates our definition of approximate symmetries as local minima of the distortion.

We therefore define approximate symmetries as

$$
\operatorname{Sym}_{\mathcal{E}}(X, d)=\left\{g \in \operatorname{Iso}_{\mathcal{\varepsilon}}(X, d): \operatorname{dis}(g, d) \leq \operatorname{dis}(f, d) \forall f \in B_{\Pi(X)}(g)\right\} .
$$

Numerically, we observed that for a small $\varepsilon$, the number of local minima is similar to the number of true symmetries of a symmetric shape.

\section{Gromov-Hausdorff distance}

An early attempt to compare shapes as metric spaces was done by Elad and Kimmel [14] who proposed to embed pairwise geodesic distances into a third fixed metric space $\left(Z, d_{Z}\right)$. Usually, a low dimensional Euclidean space is a good candidate from the computational point of view. Such a minimum distortion embedding was referred to as canonical form. It can be explicitly calculated by minimizing

$$
\min _{\varphi: X \rightarrow Z} \max _{x, x^{\prime} \in X} \mid d_{X}\left(x, x^{\prime}\right)-d_{Z}\left(\varphi(x), \varphi\left(x^{\prime}\right) \mid\right.
$$


Assuming the embedding distortion is low enough, the comparison of $\left(X, d_{X}\right)$ and $\left(Y, d_{Y}\right)$ can be reduced to rigid matching of canonical forms, e.g. using the Hausdorff distance,

$$
d_{H}^{Z}(\varphi(X), \psi(Y))=\max \left\{\max _{y \in \psi(Y)} \min _{x \in \varphi(X)} d_{Z}(x, y), \max _{x \in \varphi(X)} \min _{y \in \psi(Y)} d_{Z}(x, y)\right\}
$$

A general theoretical framework for metric spaces comparison was proposed by Gromov [9, 18], and introduced to shape analysis by Mémoli ans Sapiro [31]. Since it is generally impossible to select a common metric space $\left(Z, d_{Z}\right)$ accommodating all shapes, Gromov suggested to optimize also for $Z$, resulting in the following distance, referred to as the Gromov-Hausdorff distance,

$$
d_{G H}(X, Y)=\inf _{\substack{\varphi: X \rightarrow Z \\ \psi: Y \rightarrow Z \\ Z}} d_{H}^{Z}(\varphi(X), \psi(Y))
$$

where $\psi$ and $\varphi$ are isometric mappings. The Gromov-Hausdorff distance can be alternatively expressed in terms of correspondences between the two metric spaces [9] as

$$
d_{G H}(X, Y)=\frac{1}{2} \inf _{C} \operatorname{dis}(C),
$$


where $C \subset X \times Y$ is a correspondence between the spaces, and $\operatorname{dis}(C)$ is its distortion

$$
\operatorname{dis}(C)=\sup _{(x, y),\left(x^{\prime}, y^{\prime}\right) \in C}\left|d_{X}\left(x, x^{\prime}\right)-d_{Y}\left(y, y^{\prime}\right)\right|
$$

Note that for $C$ to be a valid correspondence, for each $x \in X$ there must exist at least one $y \in Y$ such that $(x, y) \in C$, and vice versa, for each $y \in Y$ there must exist at least one $x \in X$ such that $(x, y) \in C$.

In [5], it was shown how (17) can be efficiently approximated using a convex optimization framework. In this paper, we adopt this scheme for the computation of self-isometries.

\section{Numerical framework}

For practical computation of symmetries, the surface $X$ has to be discretized and sampled at $N$ points, constituting an $r$-covering (i.e., $X=\bigcup_{n=1}^{N} B_{X}\left(x_{n}, r\right)$, where $B_{X}$ denotes a closed ball on $X$ induced by the metric $d_{X}$ ). We denote this sampling by $X_{r}=\left\{x_{1}, \ldots, x_{N}\right\} \subseteq X$. A good sampling strategy can be achieved using the (2 optimal) farthest point sampling algorithm [20], which guarantees that $X_{r}$ is also $r$-separated, i.e. $d_{X, t}\left(x_{i}, x_{j}\right) \geq r$ for any $i \neq j$. In the following, we assume that $X$ is approximated by a triangular mesh $\hat{X}$ built upon the vertices $X_{r}$. 


\subsection{Approximation of distances}

In order to approximate the geodesic distances, we use the fast marching method [23], which produces a first-order approximation for the geodesic distances between $X_{r}$ on $\hat{X}$. The main idea of fast marching is to simulate wavefront propagation on a triangular mesh, associating the time of arrival of the front with the distance traveled, assuming constant propagation speed. A linear time is required to obtain the geodesic distance between two points $[44,45,46]$.

The diffusion metric is computed according to (6). For this purpose, we first compute the discrete approximation of the Laplace-Beltrami operator on the mesh. A Finite Elements Method (FEM) approach is possible [39], but in this work we used the following generic form

$$
\left(\Delta_{\hat{X}} f\right)_{i}=\frac{1}{a_{i}} \sum_{j} w_{i j}\left(f_{i}-f_{j}\right)
$$

where $f: \hat{X} \rightarrow \mathbb{R}$ is a scalar function defined on the mesh $\hat{X}$ and represented as a vector of function values at the vertices of the mesh, $w_{i j}$ are weights, and $a_{i}$ are normalization coefficients. In matrix notation, Equation (19) can be written as

$$
\Delta_{\hat{X}} f=A^{-1} L f
$$

where $A=\operatorname{diag}\left(a_{i}\right)$ and $L=\operatorname{diag}\left(\sum_{l \neq i} w_{i l}\right)-\left(w_{i j}\right)$.

There exists several discretizations of the Laplace-Beltrami operator [4, 48], for which $A$ and $W$ are defined. Here we adopt the cotangent weight scheme 
[36], in which $a_{i}$ is set to be half of the area of the circumcentric dual of vertex $i$, and $w_{i j}=\cot \alpha_{i j}+\cot \beta_{i j}$, where $\alpha_{i j}$ and $\beta_{i j}$ are the angles opposite to the edge between the $i$ 'th and $j$ 'th vertices, if they are connected, and zero otherwise.

By solving the generalized eigendecomposition problem [26]

$$
A \phi=\lambda L \phi
$$

the $k$ smallest eigenvalues $\lambda_{0}, \ldots, \lambda_{k}$ and corresponding eigenfunctions $\phi_{0}, \ldots, \phi_{k}$ : $\hat{X} \rightarrow \mathbb{R}$ of the discretized Laplace-Beltrami operator are computed.

\subsection{Coarse matching}

Discrete permutations on $X_{r}$ can be represented as $N$-tuples of the form $\pi=$ $\left(\pi_{1}, \ldots, \pi_{N}\right) \in\{1, \ldots, N\}^{N}$. Finding all permutations with a distortion lower than $\varepsilon$ requires computation of the distortion of $\mathscr{O}(N !)$ possible mappings. However, the search space can be greatly reduced by ruling out mappings that cannot have low distortion. We observe that for a good candidate of an approximate symmetry, the intrinsic properties of the surface, such as local intrinsic geometry around $x_{i}$ should be similar to that around $x_{\pi_{i}}$. In order to quantify this behavior, for each $x_{i} \in X_{r}$ we compute the histogram $h_{i}=\operatorname{hist}\left(\left\{\hat{d}_{i j}: \hat{d}_{i j} \leq \rho\right\}\right)$ of the approximate distances $\left(\hat{d}_{i j}=d_{X}\left(x_{i}, x_{j}\right)\right)$ in a $\rho$-ball centered at $x_{i}[16,28,37]$.

In order to reduce the number of possible permutations, we chose a small number of points using farthest point sampling strategy on diffusion distances to represent the symmetry. Experiments showed that resampling the surface starting 
from the second sampled point produces a better sampling because the location of the first point is arbitrary and its support may occlude a better one. Each point has many possible matches and clustering of the candidates is performed. Two points are in the same cluster if and only if there exists a chain of points on the surface with similar distance histograms. Earth mover's distance (EMD) [41] can be used as the distance between histograms.

For a given point $x$, we compute its histogram $h_{x}$ and find all points $y$ with a similar histogram, denoted by

$$
\mathrm{H}_{\delta}(x)=\left\{y: d\left(h_{x}, h_{y}\right) \leq \delta\right\}
$$

where $d$ is the distance between the histograms, and $\delta$ is calculated a priori according to the histogram distance between neighboring points. We perform $\varepsilon$ separation of the set $\mathrm{H}_{\delta}(x)$ to subsets $\mathrm{H}_{\delta}^{i}(x)$ according to

$$
\begin{gathered}
y, z \in \quad \mathrm{H}_{\delta}^{i}(x) \quad \text { iff } y, z \in \mathrm{H}_{\delta}(x) \text { and } \exists\left\{y_{k}\right\}_{1}^{m} \in \mathrm{H}_{\delta}(x) \\
\text { s.t. } y_{1}=y, y_{m}=z, \quad \forall 1 \leq k \leq m-1 \\
\quad d_{X}\left(y_{k}, y_{k+1}\right) \leq \varepsilon .
\end{gathered}
$$

Once possible sets are constructed, we search for a global correspondence between candidates of each set. Since the number of sets is small, the search becomes feasible. Solving (23) is done in similar spirit to union-set data structure methods, as follows: 
1. Choose one point $y$ at random.

2. Find the farthest point $q$ from it.

3. Resample the mesh using farthest point sampling starting with $q$.

4. Repeat the following stages for each point sample $x$.

(a) Calculate $\operatorname{Hist}_{\delta}(x)$.

(b) $\mathrm{i}=1 . \forall i S_{i}=\{\}$

(c) Choose a point $z \in \operatorname{Hist}_{\delta}(x) \backslash \bigcup_{k=1}^{i} S_{k}$.

(d) Set $S_{i}=\{z\}$.

(e) For each point $q \in S_{i}$ :

$$
S_{i}=S_{i} \bigcup\left\{g \in \mathrm{H}_{\delta}(x) \text { s.t. } d(q, g) \leq \varepsilon\right\}
$$

(f) repeat step (e) until convergence.

(g) if $\bigcup_{k=1}^{i} S_{k} \neq \mathrm{H}_{\delta}(x): \mathrm{i}=\mathrm{i}+1$, goto step (c)

(h) $m_{i}^{x}=\operatorname{argmin}_{q}\left\{d\left(h_{x}, h_{q}\right)\right.$ s.t. $\left.q \in S_{i}\right\}$.

Each point $x$ has $\left\{m_{i}^{x}\right\}$ set as possible matches.

In many practical problems, exhaustive search is possible. In cases where search complexity is still prohibitive, we use a branch and bound algorithm similar to [16]. While complexity remains the same, the search space becomes extremely small in practice. As presented in [37], we apply a branch and bound procedure, for $M$ points, as follows. 
1. Given a correspondence of $k-1$ feature points $(1, \ldots, k-1) \mapsto\left(\pi_{1}, \ldots, \pi_{k-1}\right)$, we would like to establish $k \mapsto \pi_{k}$.

2. Prune: For each potential correspondence $\pi_{k} \in C_{k}$, evaluate $\max _{i=1, \ldots, k} \mid \hat{d}_{i k}-$ $\hat{d}_{\pi_{i}, \pi_{k}} \mid$. If the obtained distortion is larger than $\varepsilon$, discard the potential correspondence.

3. Branch: For each remaining $\pi_{k}$, recursively invoke Step 1 with $(1, \ldots, k) \mapsto$ $\left(\pi_{1}, \ldots, \pi_{k}\right)$.

4. Bound: If $k=M$, compute the distortion $\operatorname{dis}(\pi, d)$. If $\operatorname{dis}(\pi, d)<\varepsilon$, mark $\pi$ as an $\varepsilon$-symmetry.

\subsection{Generalized MDS}

Once a coarse match is found, it is used as an initialization of the second refinement stage. Solving (17) requires to find a correspondence which distorts the metric the least. Following the discretization in [5], for $N$ mesh points $x_{i}$ on $\hat{X}$ we search for $x_{i}^{\prime}$ locations, (not necessarily coincide with the mesh vertices), with similar diffusion distances between them, minimizing

$$
\min _{x_{1}^{\prime}, \ldots, x_{N}^{\prime} \in \hat{X}} \max _{i, j=1, \ldots, N}\left|\hat{d}_{i j}-\hat{d}_{X}\left(x_{i}^{\prime}, x_{j}^{\prime}\right)\right|^{2}
$$

We use the convex optimization scheme presented in [5], referred to as generalized multidimensional scaling (GMDS), to solve (24). 


\section{Experimental results}

In order to show the advantages using diffusion distances, we performed several experiments on meshes taken from the TOSCA dataset [7]. The first coarse matching exploration stage took a couple of seconds per surface, and the second stage using GMDS refinement procedure took less than a minute for approximately 2000 points on a Pentium Core 2 Duo 3.0 GHz CPU.

Figure 1 depicts the histograms of geodesic and diffusion distances of two matching points. Without a topology change, all histograms are almost identical, while with a topology change only the diffusion histograms preserve their similarity.

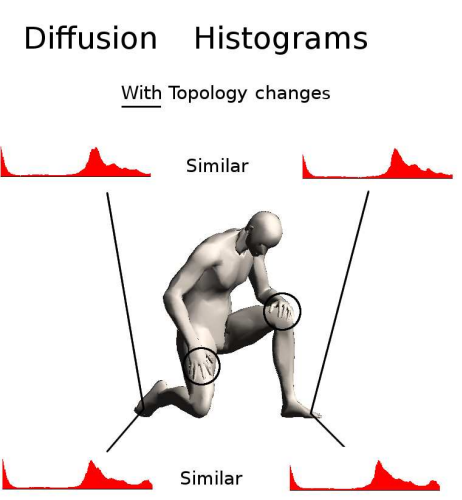

Without Topology changes

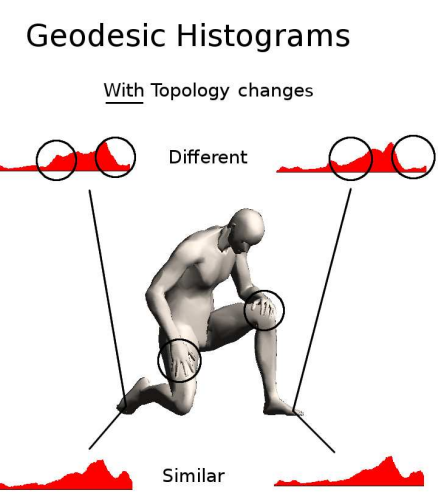

Without Topology changes

Figure 1: Geodesic and diffusion histograms of two matching sample points with and without topology changes. Black circles mark points where topology changes were introduced, and over the major non-matching parts of the histograms.

In Figure 2 we present geodesic and diffusion maps from a point source. In 
contrast to geodesic distances, diffusion distances are largely unaffected by topology changes.



Figure 2: Color coded distances from the foot (marked with an arrow) using two different metrics under topology changes. In contrast to geodesic distances, diffusion distances are hardly affected by topology changes. Black circles mark points where topology changes were introduced.

Figure 3 shows the best five coarse symmetries of a human body undergoing several topology changes. The reflective symmetry (E) is found as well as additional twists (B,C,D). In a small number of points false negatives appear, and these can be rejected using either the GMDS procedure, adding descriptors, or 
increasing the sample size.

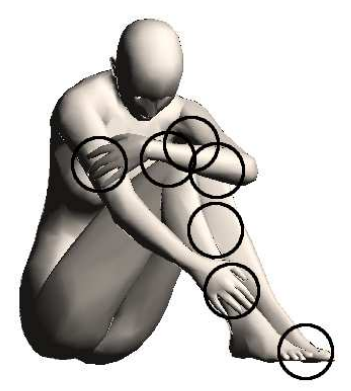

0.015351

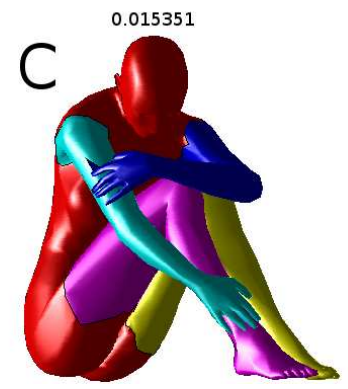

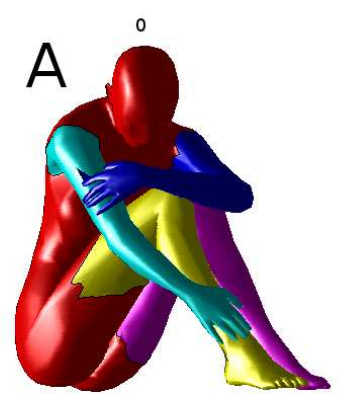

0.015654



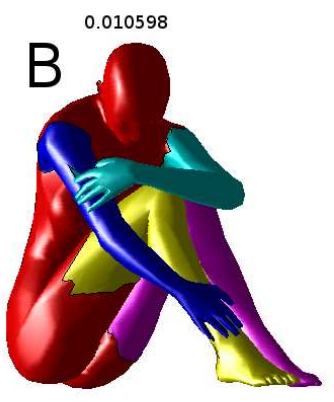

0.016473

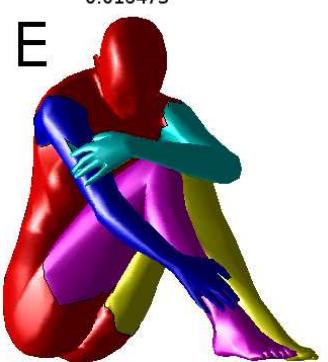

Figure 3: Coarse symmetries of a human body. Similar parts appear in similar colors, and the total distortion is indicated as a numerical value. The distortion less identity symmetry is (A). The full reflective symmetry is (E) while (B), (C), and (D) represent possible symmetries with partial reflection. Black circles mark points where topology changes were introduced.

The price paid to cope with topology changes is discriminatively. One can think of diffusion distances as an averaging process. As such it discriminates less the geometric features. From Figure 4 it is evident that the diffusion histograms of the palms, the legs and the face, are similar. Measuring the distance between the diffusion histogram descriptors alone can produce false positive matchings. Indeed, the histograms are not identical, but to cope with reasonable deformations we need to increase the thresholds, which can produce false positives. For exam- 
ple, if point 4 and 5 should be considered a match then point 3 could be a candidate as well. Such false-positives can be removed using the branch-and-bound procedure, as described earlier, or even by exhaustive search on all 5! permutations, by measuring the diffusion distances between those five points.

\section{Histogram similarity}

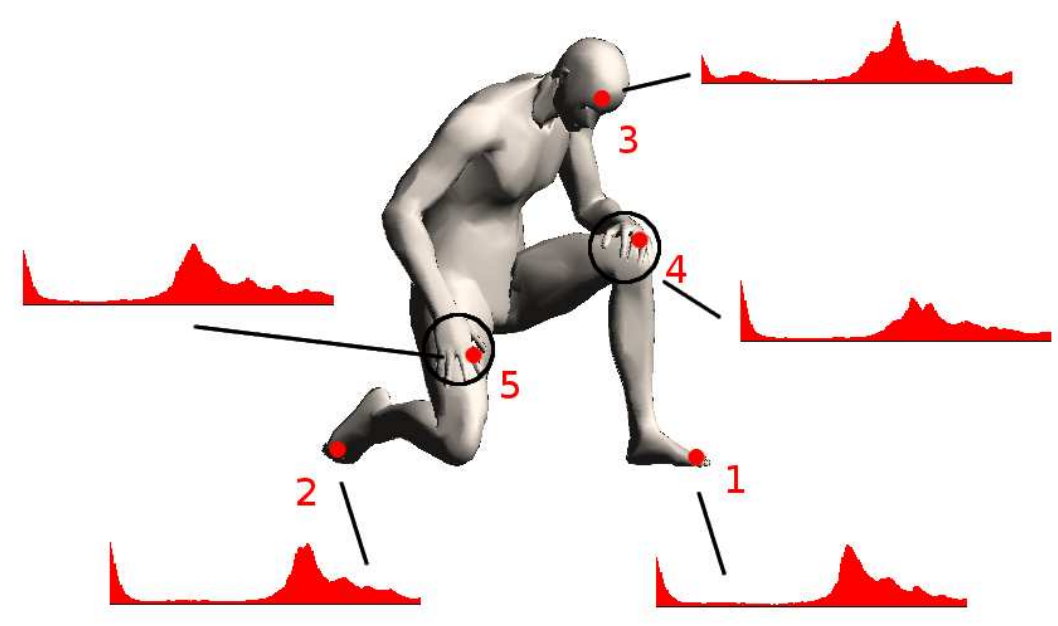

Figure 4: Diffusion histograms of different points on the body are not identical but similar due to the diffusion averaging process. A match between points 4 and 5 can inflict a false-positive match with point 3 as well. Black circles mark points where topology changes were introduced.

Figure 5 depicts symmetries constructed by using GMDS. We used $L_{2}$ instead of $L_{\infty}$ in the process, which provided better numerical results. Each blue line represents a possible match from a set of 128 matches. We show several matches 
on semi-transparent human bodies. We calculated self-matching on three different meshes with and without topology changes, of different strengths (A-C), using geodesic and diffusion distances. Four experiments were performed for each mesh, and the quality of the correspondence with and without a topology change, for geodesic and diffusion distances, was measured in terms of the geodesic distances from the ground truth. For ground truth $x_{i}$ points and $\hat{x}_{i}$ calculated positions we quantified the correspondence quality by

$$
d_{\mathscr{C}}(x, \hat{x})=\frac{\Sigma_{i} d_{X}\left(x_{i}, \hat{x}_{i}\right)}{n \cdot \operatorname{Diam}(X)},
$$

where $\operatorname{Diam}(X)$ is the diameter of $X$ and $n$ is the number of points used in the optimization stage, which produces a scaling and sampling invariant measure.

Correspondence quality is summarized in Table 1. Without topology changes both diffusion distances and geodesic distances produce good results, but with the presence of topology changes the performance of geodesic distances is highly degrades while diffusion distances keep their accuracy. For example, in mesh (C) the topology change caused a decrease in correspondence accuracy by $33 \%$ using diffusion distances, and by more than 4 times when using geodesic distances.

Figure 6 depicts the local shape asymmetry of a human body with local asymmetry introduced by deforming one of the palms followed by adding a topology change, marked with a black circle. Even though the body does not exhibit nontrivial extrinsic symmetries, we were still able to find its local intrinsic asymmetry, marked with an arrow. High asymmetry values are shaded in blue. We extracted 


\begin{tabular}{|l|l|l|l|l|}
\hline & \multicolumn{2}{|c|}{ same topology } & \multicolumn{2}{c|}{ different topology } \\
\hline & Geodesic & Diffusion & Geodesic & Diffusion \\
\hline A & 0.0091 & 0.0094 & $0.0156(71 \%)$ & $0.0126(34 \%)$ \\
\hline B & 0.0187 & 0.0125 & $0.0243(30 \%)$ & $0.0125(11 \%)$ \\
\hline C & 0.0160 & 0.0222 & $0.0901(463 \%)$ & $0.0222(33 \%)$ \\
\hline
\end{tabular}

Table 1: Correpondense quality measured by $d_{\mathscr{C}}(x, \hat{x})(25)$ on three different meshes with and without a topology change, using geodesic and diffusion distances. We added the percentage decrease in accuracy, due to topology changes, in parentheses. The meshes can be seen in Figure 5.

A
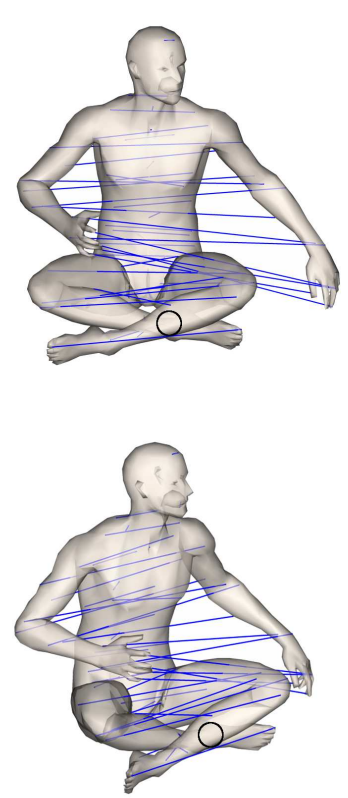

B
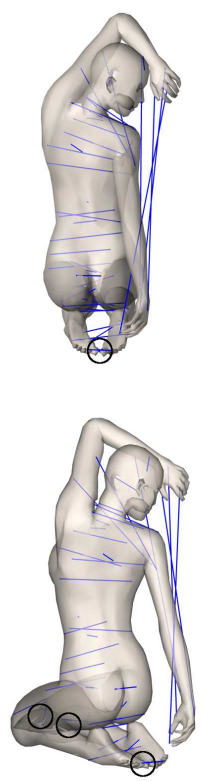

C
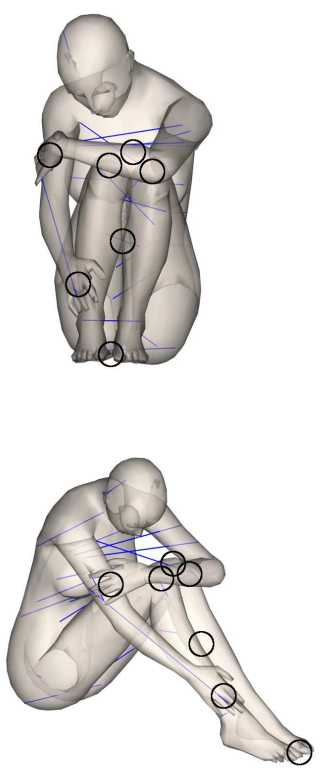

Figure 5: GMDS self-embeddings. Matching points are connected with a blue line on partial transparent human bodies. (A) poses small topology changes, (B) mediocre , and $(\mathrm{C})$ extreme ones. Black circles are drawn over connectors which caused topology changes. 
a non-trivial intrinsic symmetry that appeared as an intrinsic reflection, and then used that mapping to detect the abnormality at the palm. The method had difficulties locating asymmetric parts using geodesic distances, yet succeeded using diffusion distances.
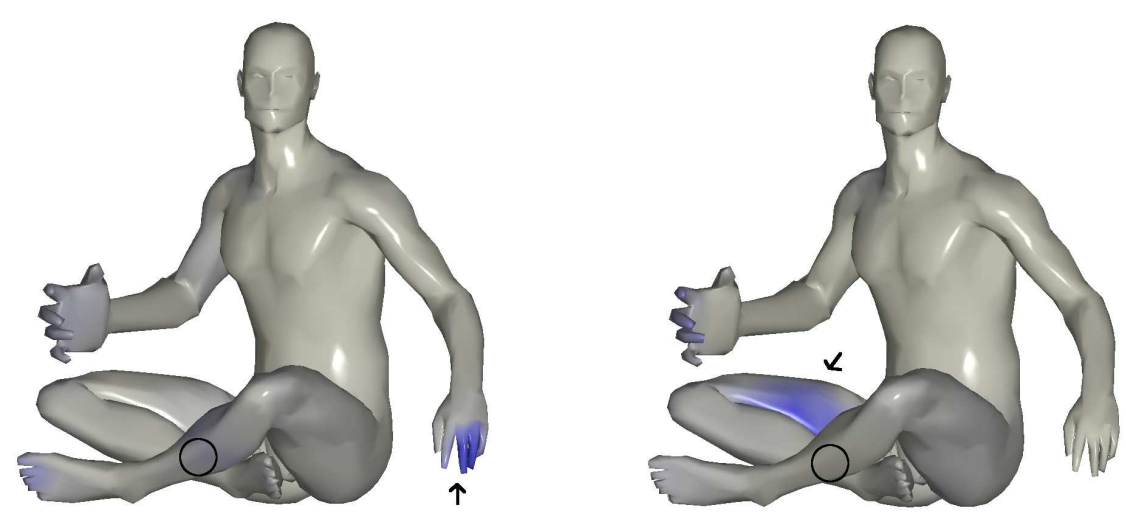

Figure 6: Calculating local asymmetry values for a possible reflective symmetry, using geodesic distances (right) and diffusion distances (left). The right palm was stretched and a topology change was inflicted on the legs. A black circle represents the inflicted topology change, and an arrow represents the high asymmetry area of the object. Blue color represents local asymmetry. The asymmetry in the palm was detected using diffusion distances (left) but could not be isolated using geodesic distances (right).

Figure 7 presents an extrinsic reflective symmetry calculated from pairs of matching points. We assumed the shape was extrinsically symmetric, and calculated the reflective plane using Principle Component Analysis (PCA) on the centers of the connecting lines between matched points. Adding a topology change rotated the reflective plane by almost 10 degrees when geodesic distances were in use, but only by one degree while using diffusion distances. 

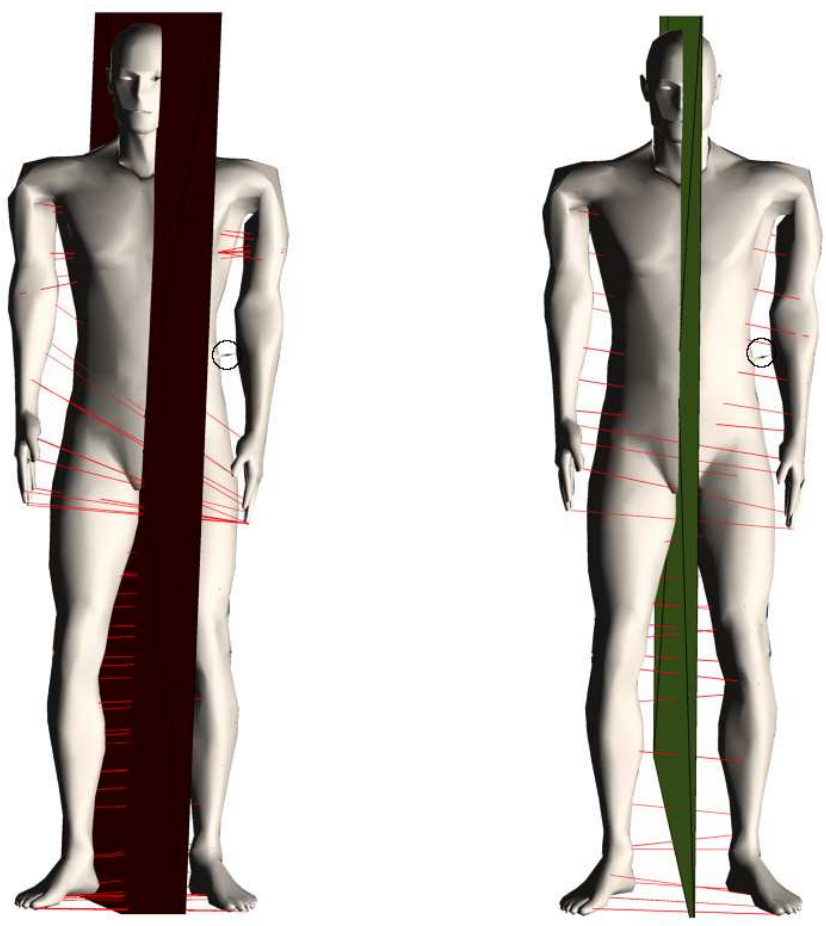

Figure 7: Extrinsic reflective symmetry calculated from matching pairs of points using geodesic (left) and diffusion (right) distances. A topology noise is marked in a black circle, and matching points by a red line. Compared to an accurate reflective plane, the topological noise rotated the plane by 9.8 degrees while using geodesic distances, and by 1.2 degrees for diffusion distances. 
For a given metric $d$, which is $t$ time depended, and a given $\varepsilon$, we can sample the space of all possible symmetries $\operatorname{Sym}_{\mathcal{E}}(X, d)$. We measure the distance between mappings according to (11), and embed this abstract space into $\mathbb{R}^{2}$ using multidimensional scaling (MDS) method.

Since the space is obviously non-Euclidean, there are distortions in the process, yet, the visual results are informative. In Figure 8 we continued sampling the symmetries of a human body from Figure 3. We computed 155 potential candidates, calculated their distortion, embedded them in $\mathbb{R}^{2}$ using classical MDS, and interpolated their distortion on a plane. We see that the symmetry space itself has one approximate reflective symmetry, indicating that a reflective symmetry is the only true symmetry of the shape. In addition, in Figure 9 we present the influence of $\varepsilon$ on the symmetry space. 


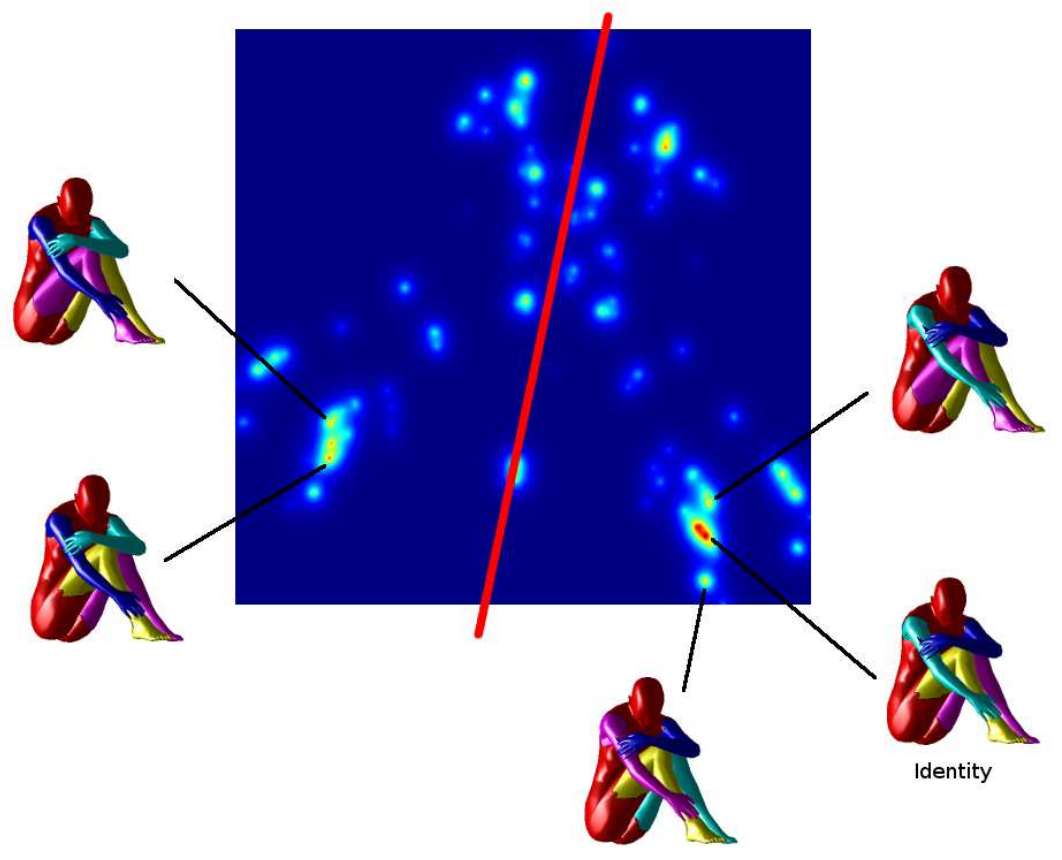

Figure 8: The symmetry space of a the human body from Figure 3 embedded in $\mathbb{R}^{2}$. Red represent low distortion embeddings (symmetries). The red line shows the reflective symmetry of the space itself. We measured the distance between functions according to (11).
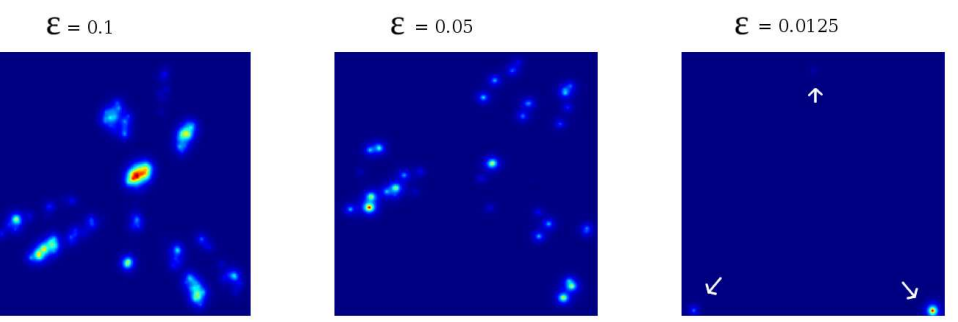

Figure 9: $\varepsilon$ influence on the symmetry space of the human body from Figure 5 embedded in $\mathbb{R}^{2}$. As $\varepsilon$ grows, more symmetries appear, but the symmetry space remains symmetric. 


\section{Conclusions}

We presented a method to extract approximate intrinsic symmetries of bendable surfaces which are topology aware, using diffusion distances. We showed how local asymmetry values can be used for detecting shape abnormalities, and presented a visualization of the entire symmetry space.

\section{Acknowledgements}

This research was supported in part by The Israel Science Foundation grant no. 623/08, and by The USA Office of Naval Research (ONR) grant. Saprio is partially supported by ARO, NGA, ONR and NSF.

\section{Appendix A}

\section{Proposition 1}

Let $g \in \Pi(X)$ be a continuous function of the metric space $\left(X, d_{X}\right)$, then $\operatorname{dis}(g, d)$ is a continuous function as well.

\section{Proof}

Since $g$ is continuous, $\forall \hat{\varepsilon} \exists \hat{\delta} \quad x, \hat{x} \in X$ such that $d_{X}(x, \hat{x}) \leq \hat{\delta} \Longrightarrow$ $d_{X}(g(x), g(\hat{x}) \leq \hat{\varepsilon}$.

In order to prove that dis is continuous it is sufficient to show that 
$\forall \varepsilon \exists \delta \quad x, \hat{x}, y, \hat{y} \in X$

$$
\begin{aligned}
& d_{X}(x, \hat{x})+d_{X}(y, \hat{y}) \leq \delta \Longrightarrow \\
& \left|\max _{x, y}\right| d_{X}(x, y)-d_{X}(g(x), g(y))\left|-\max _{\hat{x}, \hat{y}}\right| d_{X}(\hat{x}, \hat{y})-d(g(\hat{x}), g(\hat{y}))|| \leq \varepsilon
\end{aligned}
$$

But if $g$ is $\bar{\varepsilon}$-symmetry,

$$
\begin{aligned}
& \max _{x, y}\left|d_{X}(x, y)-d_{X}(g(x), g(y))\right|-\max _{\hat{x}, \hat{y}}\left|d_{X}(\hat{x}, \hat{y})-d_{X}(g(\hat{x}), g(\hat{y}))\right| \\
& \leq \max _{x, y, \hat{x}, \hat{y}}|| d_{X}(x, y)-d_{X}\left(g(x), g(y)|-| d_{X}(\hat{x}, \hat{y})-d_{X}(g(\hat{x}), g(\hat{y}))||\right. \\
& \leq \max _{x, y, \hat{x}, \hat{y}}\left|d_{X}(x, y)-d(\hat{x}, \hat{y})\right|+\left|d_{X}(g(x), g(y))-d_{X}(g(\hat{x}), g(\hat{y}))\right| \\
& \leq 2 \delta+\bar{\varepsilon}+2 \hat{\varepsilon} .
\end{aligned}
$$

Then, for a small $\hat{\varepsilon}$, and a locally small $\bar{\varepsilon}$

$$
\delta=\frac{\varepsilon-\bar{\varepsilon}-2 \hat{\varepsilon}}{2}
$$

is required for (26) to hold. Note, that $\bar{\varepsilon}$ can be infinitely small only near true symmetries. Obviously, it does not mean that near $\mathcal{\varepsilon}$-symmetry there exists an $\frac{1}{2} \varepsilon$-symmetry. 


\section{References}

[1] Alt, H., Mehlhorn, K., Wagener, H., Welzl, E.: Congruence, similarity, and symmetries of geometric objects. Discrete and Computational Geometry $\mathbf{3}$, 237-256 (1988)

[2] Atallah, M.J.: On symmetry detection. IEEE Trans. Computers c-34(7) $(1985)$

[3] Belkin, M., Niyogi, P.: Laplacian eigenmaps for dimensionality reduction and data representation. Neural Comput. 15(6), 1373-1396 (2003)

[4] Bobenko, A., Springborn, B.: A discrete laplace-beltrami operator for simplicial surfaces. Discrete and Computational Geometry 38(4), 740-756 (2007)

[5] Bronstein, A.M., Bronstein, M.M., Kimmel, R.: Efficient computation of isometry-invariant distances between surfaces. SIAM Journal on Scientific Computing 28/5, 1812-1836 (2006)

[6] Bronstein, A.M., Bronstein, M.M., Kimmel, R.: Generalized multidimensional scaling: a framework for isometry-invariant partial surface matching. Proceedings of the National Academy of Sciences (PNAS) 103(5), 1168$1172(2006)$

[7] Bronstein, A.M., Bronstein, M.M., Kimmel, R.: Numerical Geoemtry of non-rigid shapes. Springer-Verlag (2008) 
[8] Bronstein, A.M., Bronstein, M.M., Kimmel, R., Mahmoudi, M., Sapiro, G.: A Gromov-Hausdorff framework with diffusion geometry for topologicallyrobust non-rigid shape matching. International Journal of Computer Vision (IJCV) (2009)

[9] Burago, D., Burago, Y., Ivanov, S.: A course in metric geometry, Graduate studies in mathematics, vol. 33. American Mathematical Society (2001)

[10] Cheung, K., Ip, H.: Symmetry detection using complex moments. In: Proc. International Conference on Pattern Recognition (ICPR), vol. 2, pp. 14731475 (1998)

[11] Coifman, R.R., Lafon, S., Lee, A.B., Maggioni, M., Nadler, B., Warner, F., Zucker, S.W.: Geometric diffusions as a tool for harmonic analysis and structure definition of data: Diffusion maps. Proc. National Academy of Sciences 102(21), 7426-7431 (2005)

[12] Cornelius, H., Loy, G.: Detecting rotational symmetry under affine projection. In: Proc. International Conference on Pattern Recognition (ICPR), vol. 2, pp. 292-295 (2006)

[13] Derrode, S., Ghorbel, F.: Shape analysis and symmetry detection in graylevel objects using the analytical fourier-mellin representation. Signal Processing 84(1), 25-39 (2004) 
[14] Elad, A., Kimmel, R.: On bending invariant signatures for surfaces. IEEE Trans. on Pattern Analysis and Machine Intelligence (PAMI) 25(10), 1285$1295(2003)$

[15] Gal, R., Shamir, A., Cohen-Or, D.: Pose-oblivious shape signature. IEEE Transactions on Visualization and Computer Graphics 13(2), 261-271 (2007)

[16] Gelfand, N., Mitra, N.J., Guibas, L.J., Pottmann, H.: Robust global registration. In: Proc. Eurographics Symposium on Geometry Processing (SGP), pp. 197-206 (2005)

[17] Gofman, Y., Kiryati, N.: Detecting symmetry in grey level images: The global optimization approach. In: Proc. International Conference on Pattern Recognition (ICPR), pp. 951-956 (1996)

[18] Gromov, M.: Structures métriques pour les variétés riemanniennes. No. 1 in Textes Mathématiques (1981)

[19] Hamza, A., Krim, H.: Probabilistic shape descriptor for triangulated surfaces. In: Proc. IEEE International Conference on Image Processing (ICIP), vol. 1, pp. 1041-1044 (2005)

[20] Hochbaum, D., Shmoys, D.: A best possible heuristic for the k-center problem. Mathematics of Operations Research 10:2, 180-184 (1985) 
[21] Jones, P., Maggioni, M., Schul, R.: Manifold parametrizations by eigenfunctions of the laplacian and heat kernels. Proceedings of the National Academy of Sciences (PNAS) 105(6), 1803 (2008)

[22] Kazhdan, M., Chazelle, B., Dobkin, D., Funkhouser, T., Rusinkiewicz, S.: A reflective symmetry descriptor for 3D models. Algorithmica 38(1), 201-225 (2003)

[23] Kimmel, R., Sethian, J.A.: Computing geodesic paths on manifolds. Proceedings of the National Academy of Sciences (PNAS) 95(15), 8431-8435 (1998)

[24] Knossow, D., Sharma, A., Mateus, D., Horaud, R.P.: Inexact matching of large and sparse graphs using laplacian eigenvectors. In: Proceedings 7th Workshop on Graph-based Representations in Pattern Recognition, LNCS 5534 (2009)

[25] Lafon, S.: Diffusion Maps and Geometric Harmonics. Ph.D. dissertation, Yale University (2004)

[26] Lévy, B.: Laplace-Beltrami eigenfunctions towards an algorithm that "understands" geometry. In: Int'l Conf. Shape Modeling and Applications (2006)

[27] Loy, G., Eklundth, J.: Detecting symmetry and symmetric constellations of features. In: Proc. Computer Vision and Pattern Recognition (CVPR), vol. 2, pp. 508-521 (2006) 
[28] Mahmoudi, M., Sapiro, G.: Three-dimensional point cloud recognition via distributions of geometric distances. Graphical Models 71(1), 22-31 (2009)

[29] Marola, G.: On the detection of axes of symmetry of symmetric and almost symmetric planner images. IEEE Trans. on Pattern Analysis and Machine Intelligence (PAMI) 11(1) (1989)

[30] Mémoli, F.: Spectral Gromov-Wasserstein distances for shape matching. In: Workshop on Non-Rigid Shape Analysis and Deformable Image Alignment (NORDIA) (2009)

[31] Mémoli, F., Sapiro, G.: A theoretical and computational framework for isometry invariant recognition of point cloud data. Foundations of Computational Mathematics 5, 313-346 (2005)

[32] Mitra, N.J., Guibas, L.J., Pauly, M.: Partial and approximate symmetry detection for 3D geometry. In: Proc. International Conference and Exhibition on Computer Graphics and Interactive Techniques (SIGGRAPH), pp. 560568 (2006)

[33] Ovsjanikov, M., Bronstein, A.M., Bronstein, M.M., Guibas, L.: Shape google: a computer vision approach for invariant retrieval of non-rigid shapes. In: Proc. Workshop on Non-rigid Shapes and Deformable Image Alignment (NORDIA). (2009) 
[34] Ovsjanikov, M., Sun, J., Guibas, L.: Global intrinsic symmetries of shapes. In: Proc. Eurographics Symposium on Geometry Processing (SGP), vol. 27 (2008)

[35] Pauly, M., Mitra, N., Wallner, J., Guibas, H.P.L.: Discovering structural regularity in 3d geometry. In: Proc. International Conference and Exhibition on Computer Graphics and Interactive Techniques (SIGGRAPH), pp. 1-11 (2008)

[36] Pinkall, U., Polthier, K.: Computing discrete minimal surfaces and their conjugates. Experimental mathematics 2(1), 15-36 (1993)

[37] Raviv, D., Bronstein, A.M., Bronstein, M.M., Kimmel, R.: Symmetries of non-rigid shapes. In: Proc. Workshop on Non-rigid Registration and Tracking (NRTL). (2007)

[38] Raviv, D., Bronstein, A.M., Bronstein, M.M., Kimmel, R.: Full and partial symmetries of non-rigid shapes. International Journal of Computer Vision (IJCV) (2009)

[39] Reuter, M., Biasotti, S., Giorgi, D., Patanè, G., Spagnuolo, M.: Discrete laplace-beltrami operators for shape analysis and segmentation. Computers \& Graphics 33, 381-390 (2009)

[40] Reuter, M., Wolter, F.E., Peinecke, N.: Laplace beltrami spectra as shapedna of surfaces and solids. Computer-Aided Design 38, 342-366 (2006) 
[41] Rubner, Y., Tomasi, C., Guibas, L.J.: The earth mover's distance as a metric for image retrieval. International Journal of Computer Vision 40(2), 99-121 (2000)

[42] Rustamov, R.M.: Laplace-beltrami eigenfunctions for deformation invariant shape representation. In: Proc. Eurographics Symposium on Geometry Processing (SGP), pp. 225-233 (2007)

[43] Sun, J., Ovsjanikov, M., Guibas, L.: A Concise and Provably Informative Multi-Scale Signature Based on Heat Diffusion . In: Proc. Eurographics Symposium on Geometry Processing (SGP) (2009)

[44] Tsitsiklis, J.N.: Efficient algorithms for globally optimal trajectories. IEEE Transactions on Automatic Control 40(9), 1528-1538 (1995)

[45] Weber, O., Devir, Y.S., Bronstein, A.M., Bronstein, M.M., Kimmel, R.: Parallel algorithms for approximation of distance maps on parametric surfaces. ACM Trans. Graph. 27(4) (2008)

[46] Yatziv, L., Bartesaghi, A., Sapiro, G.: $\mathrm{O}(\mathrm{N})$ implementation of the fast marching algorithm. J. Computational Physics 212(2), 393-399 (2006)

[47] Zabrodsky, H., Peleg, S., Avnir, D.: Symmetry as a continuous feature. IEEE Trans. on Pattern Analysis and Machine Intelligence (PAMI) 17(12), 1154$1166(1995)$

[48] Zhang, H.: Discrete combinatorial Laplacian operators for digital geometry processing. In: SIAM Conference on Geometric Design, pp. 575-592 (2004) 